\title{
Duplications and copy number variants of 8 p23.1 are cytogenetically indistinguishable but distinct at the molecular level
}

John CK Barber ${ }^{* 1,2,3}$, Viv Maloney ${ }^{2}$, Edward J Hollox ${ }^{4}$, Annegret Stuke-Sontheimer ${ }^{5}$, Gabi du Bois ${ }^{6}$, Eva Daumiller ${ }^{6}$, Ute Klein-Vogler ${ }^{7}$, Andreas Dufke $^{7}$, John AL Armour ${ }^{4}$ and Thomas Liehr ${ }^{8}$

\footnotetext{
${ }^{1}$ Wessex Regional Genetics Laboratory, Salisbury Hospital NHS Trust, Salisbury, Wiltshire, UK; ${ }^{2}$ National Genetics Reference Laboratory (Wessex), Salisbury Hospital NHS Trust, Salisbury, Wiltshire, UK; ${ }^{3}$ Human Genetics Division, Southampton University School of Medicine, Southampton General Hospital, Southampton, UK; ${ }^{4}$ Institute of Genetics, University of Nottingham, Queen's Medical Centre, Nottingham, UK; ${ }^{5}$ Genetics Clinic, Wernigerode, Germany; ${ }^{6}$ Institute for Chromosome Diagnostics and Genetic Counselling, Boeblingen, Germany; ${ }^{7}$ Department of Medical Genetics, Eberhard-Karls University, Tuebingen, Germany; ${ }^{8}$ Institute for Human Genetics and Anthropology, Friedrich-Schiller University, Jena, Germany
}

It has been proposed that duplications of 8p23.1 are either euchromatic variants of the 8p23.1 defensin domain with no phenotypic consequences or true duplications associated with developmental delay and heart defects. Here, we provide evidence for both alternatives in two new families. A duplication of most of band 8p23.1 (circa $5 \mathrm{Mb}$ ) was found in a girl of 8 years with pulmonary stenosis and mild language delay. BAC fluorescence in situ hybridisation (FISH) and multiplex amplifiable probe hybridisation (MAPH) showed that the two copies of the duplicated segment were sited, in an alternating fashion, between three copies of a circa 300-450 kb segment from 8p23.1 distal to REPD. Copy number of the variable 8p23.1 defensin domain was consistent with duplication but within the normal range. Duplication of the GATA-binding protein 4 gene (GATA4) in this patient and others with and without heart defects, suggests it is a dosage-sensitive gene with variable penetrance. A cytogenetically similar duplication of $8 \mathrm{p} 23.1$ was found at prenatal diagnosis in a fetus, father and grandmother. There was no duplication using BAC FISH but MAPH showed 11 copies of the $360 \mathrm{~kb}$ variable defensin domain which is within the expanded range found in previous euchromatic variant carriers. Semiquantitative FISH (SQ-FISH) was consistent with a simultaneous expansion of the adjacent olfactory receptor repeats. These results distinguish duplications of 8p23.1 with clinically significant consequences from benign copy number variants, which have not yet been associated with qualitative or quantitative traits.

European Journal of Human Genetics (2005) 13, 1131-1136. doi:10.1038/sj.ejhg.5201475;

published online 3 August 2005

Keywords: duplication; triplication; 8p23.1; copy number variation; GATA4

${ }^{*}$ Correspondence: Dr J Barber, Wessex Regional Genetics Laboratory, Salisbury District Hospital, Salisbury, Wiltshire, SP2 8B], UK.

Tel: + 441722 429080; Fax: + 441722 338095;

E-mail: john.barber@salisbury.nhs.uk

Received 14 February 2005; revised 17 May 2005; accepted 29 June 2005; published online 3 August 2005
Introduction

In 1998, Barber et l $^{1}$ reported seven families in which 25 out of 27 carriers of 8 p23.1 duplications were phenotypically normal. It was proposed that duplications of $8 \mathrm{p} 23.1$ had no established phenotypic consequences and could be regarded as euchromatic variants (EVs) or cytogenetically 
visible copy number variants (CNVs). O'Malley and Storto ${ }^{2}$ and Begleiter et $\mathrm{al}^{3}$ reported two further families in support of this view and Gibbons et $a l^{4}$ found only minimal phenotypic features in three carriers from a single family. Hollox et $a l^{5}$ used multiplex amplifiable probe hybridisation (MAPH) and semiquantitative fluorescence in situ hybridisation (SQ-FISH) to show that the underlying basis of the duplication in three EV families was increased copy number of a domain containing three defensin genes and olfactory receptor (OR) repeats. Total defensin copy number in normal controls varied between two and seven while EV carriers had between nine and twelve copies.

In contrast, Kennedy et $a l^{6}$ reported a cytogenetically similar duplication of 8p23.1 in a developmentally normal girl of 16 years with a severe congenital heart defect. The authors proposed that her duplication interrupted the GATA-binding protein 4 gene (GATA4) which maps to 8p23.1 and is known to give rise to heart defects when deleted. In addition, Kondoh et $\mathrm{al}^{7}$ reported a de novo 8p23.1 duplication in an RSK2 mutation negative patient with features of Coffin-Lowry syndrome and Tsai et al ${ }^{8}$ reported a series of eight families with 8 p23.1 duplications and claimed a 'preferential' association between 8p23.1 duplications, developmental delay and congenital heart disease.

Here, we have used BAC FISH and MAPH to show that cytogenetically indistinguishable duplications of 8p23.1 may be separated into two distinct groups.

\section{Methods}

Chromosome preparation and FISH were carried out using standard techniques. Probes spanning 8p23.3 to 8p21.2 were chosen from the Ensembl $1 \mathrm{Mb}$ cloneset or tiling path (www.ensembl.org/homo_sapiens/cytoview) including one probe from 8p23.3 (RP11-338B22), five probes from 8p23.2 (RP11-336N16, RP11-45M12, RP11-16H11, RP11-29A2, and RP5-991O23), three probes from 8p23.1 (CTD-2629I16, RP11-211C9, and RP11-589N15), two probes from 8p22 (RP11-433L7 and RP11-809L8) and one probe each from 8p21.3 (RP11-369E15) and 8p21.2 (RP11-14I17). The subtelomeric probes $2205 \mathrm{a} 2$ and $2053 \mathrm{~b} 3^{9}$ and the centromeric probe pJM128 were also used. For continuity with previously reported cases, ${ }^{5}$ we used BAC 51D11 (CITB 978SK) which, as described by Trask et al, ${ }^{10}$ hybridises to a subset of OR loci including those in 8p23.1 and gives enhanced signal strength in families with 8p23.1 EVs. ${ }^{5}$ MAPH and SQ-FISH were carried out using the same methods and probe set described by Hollox et al. ${ }^{5}$

\section{Results}

Family 1

This girl was delivered after a normal pregnancy and is the only child of phenotypically and intellectually normal parents with no significant family history. At the age of 8 years, she was referred with pulmonary stenosis, height and head circumference $>97$ th centile, difficulties with concentration, perception and response, sensitivity to noise and mild language delay. She had slightly deep set eyes, hypertelorism (outer canthal distance $10 \mathrm{~cm}$, inner canthal distance of $3.5 \mathrm{~cm}$ and interpupillary distance of $6.5 \mathrm{~cm}$ ), prominent brow ridges, long arched eyebrows, a short upturned nose with a broad nasal bridge and nasal tip, a long well-demarcated philtrum and full lips (Figure 1).

A duplication of 8p23.1 was found on G-banding at the 550 band level (Figure 2a). The cytogenetic appearance of this duplication, with a fine G-dark band at the centre of an expanded G-light 8p23.1 band, was indistinguishable from previously reported $\mathrm{EVs}^{1}$ and duplications of $8 \mathrm{p} 23.1 .^{8}$ Dual-colour FISH with pairs of differentially labelled probes showed that the distal part of 8p23.1 was triplicated (probe 2629I16) and that most of the rest of band $8 \mathrm{p} 23.1$ was directly duplicated (Figure $3 \mathrm{a}-\mathrm{c}$ ) including the interval between the OR repeats (probes 211C9 and 589N15). The two copies of the duplicated segment alternated between the three copies of the triplicated segment (Figures $3 \mathrm{a}-\mathrm{c}$ and 4). All the other clones and sub-telomeric probes for $8 \mathrm{p}$ and $8 \mathrm{q}$ gave normal results. On the normal chromosome 8 ,
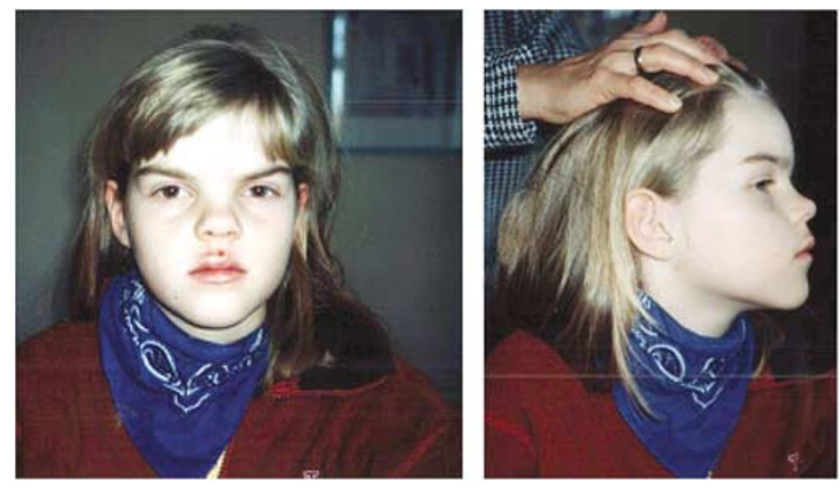

Figure 1 Images of the proband from family 1.

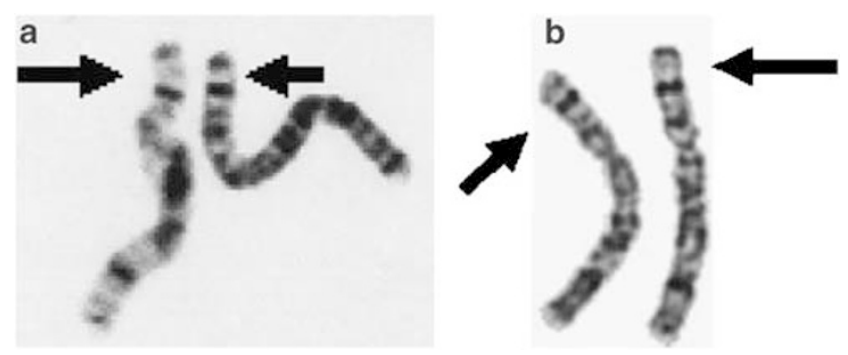

Figure 2 (a) Partial karyotypes of the proband of family 1 and (b) the grandmother from family 2 . The larger arrows point to the dup(8)trp(8) and EV chromosomes 8 and the small arrows to the normal chromosomes 8 in each case. 

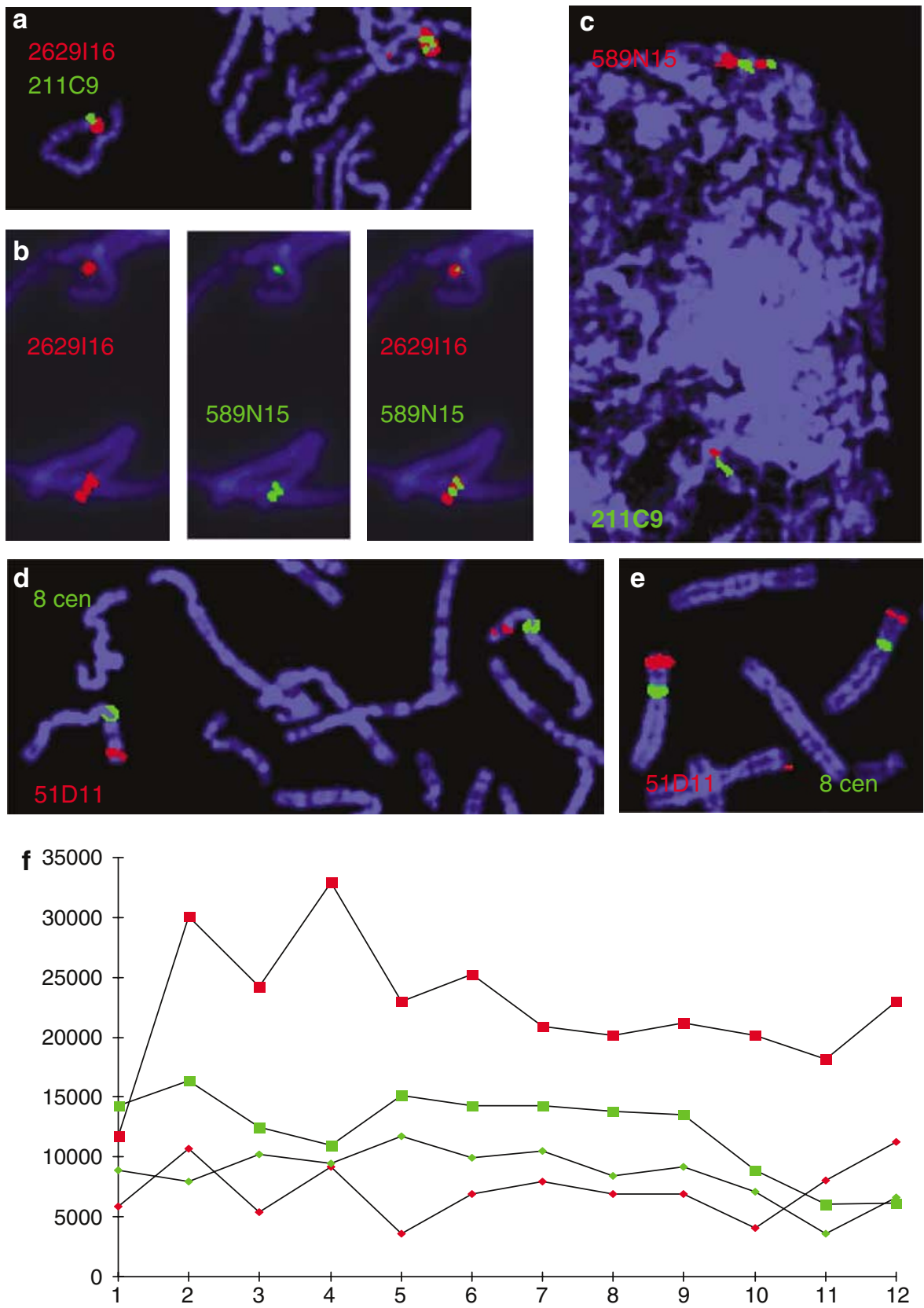

Figure 3 (a-d) Dual-colour FISH on metaphases from the proband in family 1 showing: (a) the triplication of distal 8p23.1 (red 2629116 signals) and duplication of the distal REPP-REPD interval probe (green 211C9); (b) triplication of distal 8p23.1 (red 2629116 signals in left hand panel), duplication of the proximal REPP-REPD interval probe (green 589N15) on the central panel and the combined image in the right hand panel; (c) prophase cell showing the direct duplication of both the REPP-REPD interval probes (211C9 green and 589N15 red); (d) duplication rather than triplication indicated by the separated OR BAC 51D11 signals on the dup(8)trp(8) chromosome. (e, $\mathrm{f}$ ) Dual-colour FISH on metaphases from the father in family 2 showing: (e) the contrast in signal strength with the OR BAC 51D11 between the EV and normal chromosomes and (f) a chart of the signal strengths in arbitrary fluorescent units in 12 metaphases. The large red squares represent the 51D11 signals from the EV chromosome, the large green squares the centromeric signals from the same chromosome; the small red diamonds represent the 51D11 and the small green diamonds the centromeric signals from the normal homologue.

the order of clones 211C9 and 589N15 between REPP and REPD was reversed, consistent with the common inversion found in one in four normal individuals. ${ }^{11}$
The karyotype of the proband was: $46, \mathrm{XX}, \operatorname{dup}(8)$ (p23.1p23.1)de novo. ish $\operatorname{dir} \operatorname{dup}(8)(\mathrm{p} 23.1 \mathrm{p} 23.1) \operatorname{trp}(8)$
(p23.1p23.1)
$(2205 \mathrm{a} 2+, 338 \mathrm{~B} 22+, 336 \mathrm{~N} 16+, 16 \mathrm{H} 11+$, 


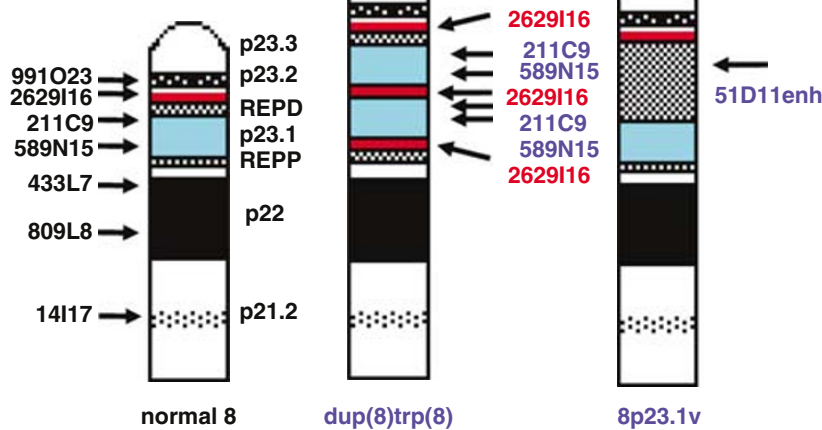

Figure 4 Partial idiograms of the chromosome $8 \mathrm{ps}$ in families 1 and 2 showing from telomere to centromere: the order of the informative probes used on the normal (left hand) homologue, the area of distal 8 p23.1 to which probe 2629116 maps (red), the position of the distal 8p23.1 OR repeat (REPD) (chequered), the interval between the REPD and REPP (blue) and the proximal 8p23.1 OR repeat (REPP) (chequered). The middle idiogram shows the inferred order of probes on the dup(8)trp(8) chromosome from family 1 and the right hand idiogram the expansion of the OR containing clone $51 \mathrm{D} 11$ on the EV chromosome from family 2.
$991 \mathrm{O} 23+, 2629 \mathrm{I} 16+, 51 \mathrm{D} 11+, 211 \mathrm{C} 9+, 589 \mathrm{~N} 15+, 2629 \mathrm{I} 16+$, $211 \mathrm{C} 9+, 589 \mathrm{~N} 15+, 2629 \mathrm{I} 16+, 51 \mathrm{D} 11+, 433 \mathrm{~L} 7+, 809 \mathrm{~L} 8+$, $14 \mathrm{I} 17+, \mathrm{pJM} 128+, 2053 \mathrm{~b} 3+)$. Both parents had normal G-banded karyotypes with no evidence of a duplication using clones 211C9 and 589N15. The mother was heterozygous and the father homozygous for the common inversion between OR repeats REPD and REPP. ${ }^{11}$ Molecular analysis confirmed that the $\operatorname{dup}(8) \operatorname{trp}(8)$ was of maternal origin (data not shown).

Total copy number across band $8 \mathrm{p} 23.1$ was estimated using MAPH (Figure 5). The results were consistent with triplication of the distal $8 \mathrm{p} 23.1$ probes $\mathrm{C}$ and $\mathrm{D}$ and with duplication of probe $\mathrm{E}$ (DEFA1) as well as probes $\mathrm{I}$ to $\mathrm{M}$ (GATA4) spanning the interval between the variable defensin domain and REPP (Figure 5). Normal results were obtained with probes $\mathrm{A}$ and $\mathrm{B}$ (from the ANGPT2 region) and probe $\mathrm{N}$ from the DCL1 locus beyond REPP.

Taken together, the FISH and MAPH results place the distal triplication breakpoint within the $160 \mathrm{~kb}$ interval between ANGPT2 (6.3-6.4 Mb) and AGPAT5 (6.55-6.6 Mb) approximately $1 \mathrm{Mb}$ distal to REPD $(7.46-7.56 \mathrm{Mb})$

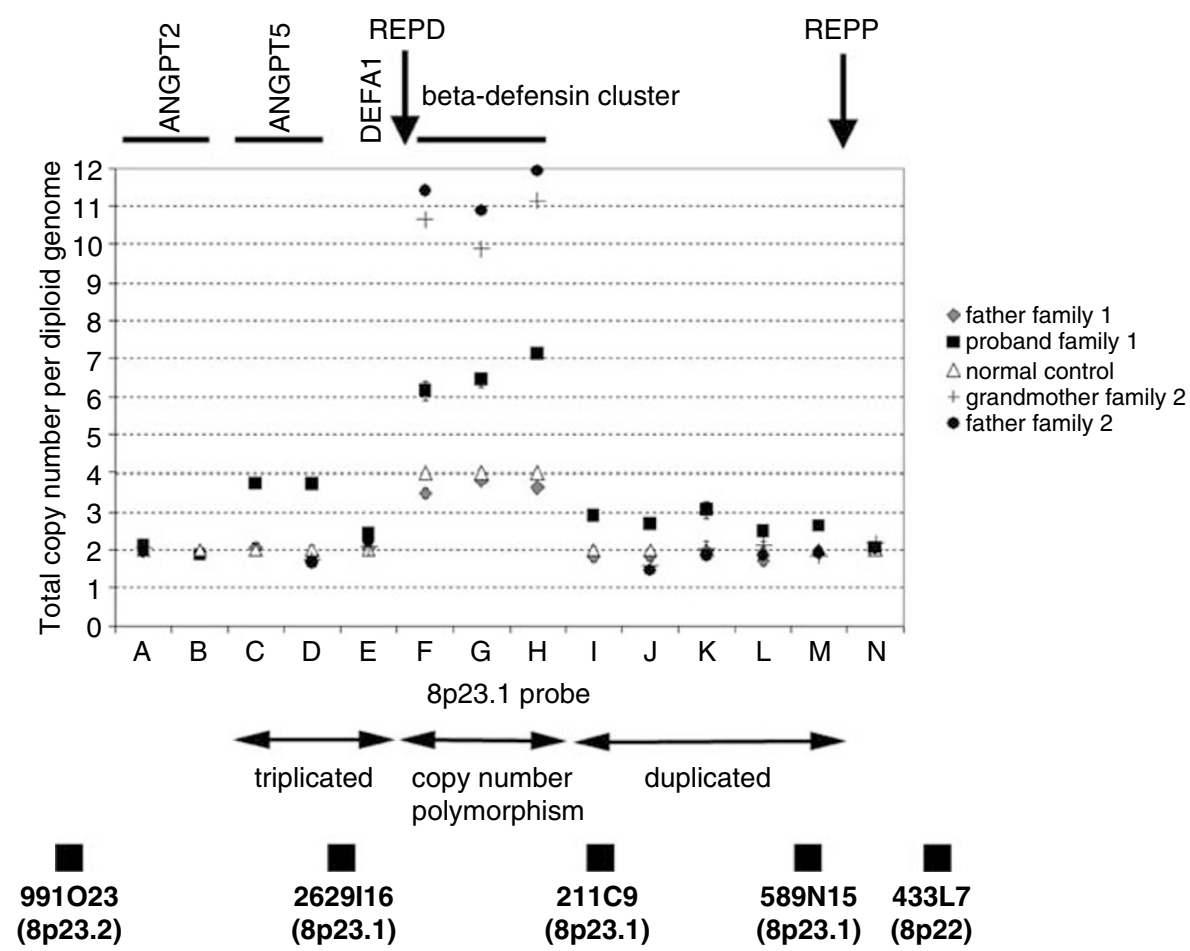

Figure 5 Summary of MAPH results across 8 p23.1 for the proband (filled squares) and father (shaded diamonds) from family 1 , the father (filled discs) and grandmother ( + signs) from family 2 and a normal control (open triangles). The mother in family 1 gave the same results as the father (data not shown). The $X$-axis shows the MAPH probes mapping to 8p23.1, distal to proximal but not to scale. Probe A maps to ANGPT2, B proximal to ANGPT2, C to FLJ11210, D between DEFB1 and DEFA4, E to DEFA1, F to DEFB4, $G$ to DEFB4, $\mathrm{H}$ to SPAG11, I to an anonymous region between SPAG1 1 and MASL1, I distal to MASL1, $\mathrm{K}$ to MASL1, L adjacent to D8S550, M to GATA4, and N to DLC1 proximal to REPP. Further details are given in Table 1 of Hollox et al. ${ }^{5}$ Probe $\mathrm{E}$ and probes $\mathrm{F}, \mathrm{G}$, and $\mathrm{H}$ report independent copy number variations. ${ }^{5}$ The blocks beneath the $X$-axis show the approximate positions of the informative BAC FISH probes. The $Y$-axis shows total copy number per diploid genome (two is normal dosage, three indicates a duplicated region and four indicates a triplicated region). Data points reflect mean and $95 \%$ confidence intervals for four replicate tests. Data for the individuals from both families were normalised against the normal control, which had a known beta-defensin copy number of four. 
(Figure 5). The boundary between the proximal triplication breakpoint and the duplication lies within the $178 \mathrm{~kb}$ interval between BAC 2629I16 and MAPH probe E (DEFA1). The proximal duplication breakpoint lies within the $\sim 1 \mathrm{Mb}$ interval between BAC 589N15 (proximal to GATA4) and MAPH probe $\mathrm{N}$ (DLC1). This interval contains REPP.

MAPH analysis also showed that the copy number of the variable defensin domain was six in the proband, five in the mother and four in the father (Figure 5). As a copy number of two per chromosome is the most common in normal individuals, ${ }^{5}$ the copy number of six in the proband is consistent with duplication but within the normal range.

\section{Family 2}

A 34-year-old woman was referred for prenatal diagnosis for advanced maternal age. The family history was unexceptional and the course of the pregnancy uneventful. Fetal ultrasound at 15 weeks gestation and follow-up examinations were normal. On G-banding at the 450 band level, an apparent duplication of 8p23.1 was found in the amniotic fluid cells, but the same abnormality was also found in father (Figure 2b) and grandmother who were both phenotypically normal. A phenotypically normal girl was born at 36 weeks 2 days gestation by caesarean resection due to breech presentation. Her birth weight was $2.975 \mathrm{~kg}$ (50th centile), birth length $50 \mathrm{~cm}$ (50th centile) and her head circumference (HC) $33 \mathrm{~cm}$ (50th centile). The postnatal course has been uneventful with growth and motor development in the normal range. At 8 weeks of age, her weight $(5.3 \mathrm{~kg})$, length $(58 \mathrm{~cm})$ and $\mathrm{HC}$ $(40 \mathrm{~cm})$ are on the 75 th centile. She has a small capillary haemangioma $(0.5-1 \mathrm{~cm}$ in diameter) on her left wrist but no other phenotypic abnormalities or minor dysmorphisms. A previous healthy 2-year-old daughter was also delivered after breech presentation at 38 weeks gestation. A normal karyotype was found by another laboratory after amniocentesis in this earlier pregnancy at the mother's request. No evidence of an abnormality of chromosome 8 was found on retrospective analysis in view of the findings reported here.

In the father, there was no duplication using FISH with any of the Ensembl BACs or the subtelomeric probes (data not shown). Enhanced (enh) signal strength was seen on one chromosome 8 using FISH with the OR BAC 51D11 (Figure 3e) and SQ-FISH gave a log adjusted ratio between the 51D11 signals on the EV and normal chromosomes of 3.20 (CI 2.55-4.03) (Figure 3f). In all but one cell, the larger 51D11 signal was consistently associated with a larger eight centromere signal (Figure 3f) and the corresponding ratio for the centromeric signals was 1.4 (CI 1.22-1.6).

The karyotype of the father was: $46, \mathrm{XY}, 8 \mathrm{p} 23.1 \mathrm{v}$.ish $8 \mathrm{p} 23.1 \mathrm{v}(2205 \mathrm{a} 2+, 45 \mathrm{M} 12+, 29 \mathrm{~A} 2+, 2629 \mathrm{I} 16+, 51 \mathrm{D} 11 \mathrm{enh}$, $211 \mathrm{C} 9+, 589 \mathrm{~N} 15+, 433 \mathrm{~L} 7+, 809 \mathrm{~L} 8+, 369 \mathrm{E} 15+, 2053 \mathrm{~b} 3+)$.
MAPH indicated that a total of 11 copies of the variable defensin domain were present in the carrier father and grandmother compared with four in the mother (Figure 5). As a copy number of two is the most common in control chromosomes, ${ }^{5}$ this implies that the EV chromosome contains 9 copies. A total of 11 copies is within the range of $9-12$ copies found in previous EV families. ${ }^{5}$

\section{Discussion}

It is remarkable the $\operatorname{dup}(8) / \operatorname{trp}(8)$ in family 1 so closely resembles the EVs in previously reported families ${ }^{1}$ and the present family 2 . They do, however, clearly differ at the molecular level as all the Ensembl 8p23.1 FISH probes, which were duplicated or triplicated in family 1 gave normal results in family 2 . In addition, MAPH showed that total copy number of the defensin domain within 8p23.1 was within the normal 2-7 range in family 1 and within the expanded 9-12 range in the adult carriers from family $2 .^{5}$ The size of the $\operatorname{dup}(8) / \operatorname{trp}(8)$ imbalance can be estimated as a minimum of $5.2 \mathrm{Mb}$ including the duplicated segment of $4.6 \mathrm{Mb}$ between REPP and REPD and the two extra copies of the $300-450 \mathrm{~kb}$ triplicated segment distal to REPD. Each of the two copies of the defensin domain which flank REPD has a minimum size of $360 \mathrm{~kb} .^{12}$ The expansion in family 2 would, therefore, span at least $3.2 \mathrm{Mb}$ assuming nine copies are present on the variant eight. In addition, the FISH results are consistent with an expansion of the OR repeats. However, repeats of the OR7E family are part of the REPD gap in the human genome sequence and we have previously been unable to estimate the size of the expansion using pulse field gel electrophoresis. ${ }^{5}$ Although the triplication breakpoints in family 1 are distal to REPD and the proximal duplication breakpoint has not yet been mapped within REPP, it is possible that the $\operatorname{dup}(8) \operatorname{trp}(8)$ chromosome represents another complex OR mediated rearrangement of $8 \mathrm{p} .^{11}$

We are not aware of an exact precedent for the duplication and triplication of 8p23.1 found in family 1 but there are at least four classes of overlapping duplications. Firstly, duplications confined to 8 p23.1 have been associated with a wide variety of presentations including developmental delay and heart disease. ${ }^{6,8}$ However, the content of these imbalances has not yet been determined and it is possible that bias of ascertainment may account for some of the affected individuals. Further FISH analysis might identify EVs as well as genuine duplications among these reported cases. Secondly, larger duplications of 8p21.3-p23.1 have been associated with developmental or speech delay ${ }^{13,14}$ and, in the proband of family 1 of Fan et $a l,{ }^{13}$ a complex heart defect. However, the mother and a sibling with the same duplication had no heart defects. Thirdly, overlapping duplications of $8 \mathrm{p} 22-\mathrm{p} 23.1$ have been reported in patients with Kabuki syndrome ${ }^{15}$ but 
these findings have not been replicated by others in clinically well characterised patients. ${ }^{16}$ Fourthly, duplications of $8 \mathrm{p} 23.1-8 \mathrm{p} 23.3$ have been reported in normal individuals ${ }^{17,18}$ and it is therefore possible that the clinical effect of the smaller triplication in family 1 is minimal. Many of these duplications include the GATA4 gene, deletions ${ }^{19}$ and intragenic mutations of which give rise to heart disease. ${ }^{20} \mathrm{~A}$ second heart disease locus has also been mapped to a $5 \mathrm{cM}$ region overlapping proximal $8 \mathrm{p} 23.1 \mathrm{but}$ a second candidate gene has not yet been identified. ${ }^{21,22}$ Taken together, these results suggest that GATA4 and/or this other locus are dosage-sensitive genes with variable penetrance.

In conclusion, these results provide the means of distinguishing cytogenetically similar duplications from copy number variants of $8 \mathrm{p} 23.1$. Our results are analogous to those in proximal $15 \mathrm{q}$ and $16 \mathrm{p}$ where constitutional cytogenetic amplification of pseudogene clusters can mimic the appearance of genuine duplications. ${ }^{23,24}$ Gains and losses near REPD are already being reported in array CGH experiments and form part of the unprecedented degree of large scale copy number polymorphism ${ }^{25,26}$ that is being collected in the Database of Genomic Variants (http://projects.tcag.ca/variation/). Further characterisation using FISH or molecular analysis is essential to determine the clinical significance of apparent duplications of $8 \mathrm{p} 23.1$.

\section{Acknowledgements}

We are indebted to the patients who contributed additional samples and photographs. We are grateful to the Sanger Centre for providing the Ensembl tiling path clones and to Dr Hiroaki Shizuya for kindly providing BAC 51D11. VM is supported as part of the National Genetics Reference Laboratory (Wessex) by the UK Department of Health Genetics, Embryology and Assisted Conception Unit and TL was supported in parts by the Dr Robert Pfleger-Stiftung fund. EJH is supported by a Welcome Trust Bioarchaeology Postdoctoral Fellowship (Grant no. 071024). The image enhancement equipment used for parts of this work was provided by the Wellcome Trust and Trust Funds of Salisbury NHS Health Care Trust. Finally, we thank Simon Thomas for his constructive reading of the manuscript.

\section{URLS}

Ensembl tiling path (www.ensembl.org/homo_sapiens/cytoview), Database of Genomic Variants (http://projects.tcag.ca/variation/)

\section{References}

1 Barber JCK, Joyce CA, Collinson MN et al: Duplication of 8p23.1: a cytogenetic anomaly with no established clinical significance. J Med Genet 1998; 35: 491-496.

2 O'Malley DP, Storto PD: Confirmation of the chromosome 8p23.1 euchromatic duplication as a variant with no clinical manifestations. Prenat Diagn 1999; 19: 178-185.

3 Begleiter ML, Cooper HA, Paszfor LM, Butler MG: Chromosome 8p23.1 duplication: is there an association with a clinical phenotype? Am J Hum Genet 2000; 65: A158,858.

4 Gibbons B, Tan SY, Barber JCK et al: Duplication of $8 \mathrm{p}$ with minimal phenotypic effect transmitted from a mother to her two daughters. J Med Genet 1999; 36: 419-422.
5 Hollox EJ, Armour JAL, Barber JCK: Extensive normal copy number variation of a $\beta$-defensin antimicrobial gene cluster. $A m$ J Hum Genet 2003; 73: 591-600.

6 Kennedy SJ, Teebi AS, Adatia I, Teshima I: Inherited duplication, $\operatorname{dup}(8)(\mathrm{p} 23.1 \mathrm{p} 23.1)$ pat, in a father and daughter with congenital heart defects. Am J Med Genet 2001; 104: 79-80.

7 Kondoh T, Takano J, Sugawara H et al: Clinical manifestations of Coffin-Lowry syndrome associated with de novo 8p23 duplication. Am J Hum Genet 2001; 69: A646.

8 Tsai C-H, Graw SL, McGavran L: 8p23 duplication reconsidered: is it a true euchromatic variant with no clinical manifestation? J Med Genet 2002; 39: 769-774.

9 National Institutes of Health and Institute of Molecular Medicine Collaboration: A complete set of human telomeric probes and their clinical application. Nat Genet 1996; 14: 86-89.

10 Trask BJ, Massa H, Brand-Arpon V et al: Large multi-chromosomal duplications encompass many members of the olfactory receptor gene family in the human genome. Hum Mol Genet 1998; 13: 2007-2020.

11 Giglio S, Broman KW, Matsumoto N et al: Olfactory receptor-gene clusters, genomic-inversion polymorphisms, and common chromosome rearrangements. Am J Hum Genet 2001; 68: 874-883.

12 Taudien S, Galgoczy P, Huse K et al: Polymorphic segmental duplications at 8 p23.1 challenge the determination of individual defensin gene repertoires and the assembly of a contiguous human reference sequence. BMC Genomics 2004; 10: 92.

13 Fan Y-S, Siu VM, Jung JH, Farrell SA, Cote GB: Direct duplication of 8p21.3-p23.1: a cytogenetic anomaly associated with developmental delay without consistent clinical features. Am J Med Genet 2001; 103: 231-234.

14 Engelen JJM, de Die-Smulders CEM, Sijstermans JMJ, Meers LEC, Albrechts JCM, Hamers AJH: Familial partial trisomy $8 \mathrm{p}$ without dysmorphic features and only mild mental retardation. $J$ Med Genet 1995; 32: 792-795.

15 Milunsky JM, Huang XL: Unmasking Kabuki syndrome: chromosome $8 \mathrm{p} 22-8 \mathrm{p} 23.1$ duplication revealed by comparative genomic hybridisation and BAC-FISH. Clin Genet 2003; 64: 509-516.

16 Miyake N, Harada N, Shimokawa O et al: On the reported 8p22p23.1 duplication in Kabuki make-up syndrome (KMS) and its absence in patients with typical KMS. Am J Med Genet 2004; 128A: $170-172$.

17 Engelen JJM, Moog U, Evers JLH, Dassen H, Albrechts JCM, Hamers AJH: Duplication of chromosome region 8p23.1> p23.3: a benign variant? Am J Med Genet 2000; 91: 18-21.

18 Harada N, Takano J, Kondoh et al: Duplication of 8p23.2: a benign cytogenetic variant? Am J Med Genet 2002; 111: 285-288.

19 Pehlivan T, Pober BR, Brueckner $\mathrm{M}$ et al: GATA4 haploinsufficiency in patients with interstitial deletion of chromosome region 8p23.1 and congenital heart disease. Am J Med Genet 1999; 83: 201-206.

20 Garg V, Kathiriya IS, Barnes R et al: GATA4 mutations cause human congenital heart defects and reveal an interaction with TBX5. Nature 2003; 424: 443-444.

21 Devriendt K, Matthijs G, Van Dael R et al: Delineation of the critical deletion region for congenital heart defects on chromosome 8p23.1. Am J Hum Genet 1999; 64: 1119-1126.

22 Giglio S, Graw SL, Gimelli G et al: Deletion of a 5-cM region at chromosome $8 \mathrm{p} 23$ is associated with a spectrum of congenital heart defects. Circulation 2000; 102: 432-437.

23 Barber JCK, Cross IE, Douglas F, Nicholson JC, Moore KJ, Browne CE: Neurofibromatosis pseudogene amplification underlies euchromatic cytogenetic duplications and triplications of proximal 15q. Hum Genet 1998; 103: 600-608.

24 Barber JCK, Reed CJ, Dahoun SP, Joyce CA: Amplification of a pseudogene cassette underlies euchromatic variation of $16 \mathrm{p}$ at the cytogenetic level. Hum Genet 1999; 104: 211-218.

25 Sebat J, Lakshmi B, Troge J et al: Large-scale copy number polymorphism in the human genome. Science 2004; 305: 525-528.

26 Iafrate AJ, Feuk L, Rivera MN et al: Detection of large-scale variation in the human genome. Nat Genet 2004; 36: 949-951. 